\title{
The Semiotics of Photography: Towards Objective Hermeneutics
}

\author{
Salvador Leon \\ Superior School of Visual Arts
}

\begin{abstract}
How can we know what a photograph means? What messages or codes exist semiotically that help us to interpret it? The mass consumption of images is reflected in the large number of contests, scholarships, galleries, museographic exhibitions and especially in advertising: What are the semiotic parameters that can be established for a reliable and clear judgment to discriminate one photo from another? For critics or judges, artists or the general public, this article seeks to answer these questions by proposing a semiotic analysis based on new advances in science, in psychology of perception and neurobiology of vision; said methodology, currently being applied intensively at ESAV and used in the postgraduate studies of the present author, seeks to facilitate the understanding of the meaning — to improve the hermeneutics — of the photographic image in a systematic and objective way.
\end{abstract}

Keywords: semiotics, semiology, arsology, photography, art, design

\section{Introduction}

What makes a configuration come to mean something to someone? How do I know what the photograph I'm perceiving means? Why facing certain images do I feel scared and with others tranquility? Can I anticipate-or even manipulate-how people will react and understand the photographic image? Is the emotional experience on the same photo, arbitrary, and absolutely subjective for each person?

For hundreds of years, humans have considered these issues, but only in the last hundred years or so, have proposals been developed to solve them: They have classified signs, analyzed the language, raised hermeneutics of art, design, and advertising. In general, this has been called Semiology or Semiotics. But although these terms are ancient—and the necessity of such a discipline was announced, semiological science was in fact practically not born until recent times.

Our object of study is hermeneutics in photography-interpretation, but the semiological one; not philosophy or sociology, but Semiology as a scientific approach. Our problem is: How do you interpret a photograph? How do I know what a photograph means? To answer these questions, we explain the method of semiotic analysis used for more than 15 years at the ESAV (School of Higher Studies on Visual Arts) where this author works; it may be added that implementing this method earned him an honorary mention, on his master's degree in Arts by the University of Guanajuato, and has helped him greatly for the development of his doctoral thesis.

Thanks to modern Neuroscience, with its interdisciplinary character and the development of experimental psychology by the American Psychological Association (AAP), along with the Institute of Color-among other scientific associations, we have been provided with more sophisticated tools that help us understand how we

Salvador Leon, Professor and photography graduate department head, Superior School of Visual Arts, ESAV, Tijuana, Mexico; main research field: Semiotics of the Photography Image. 
react to typical configurations. There are techniques of measurement that have shed light on what we feel facing different visual stimuli of the environment, applied to the experience of the perception of art (Sargent-Pollock \& Konecni 1977; Kawabata \& Zeki 2004). Techniques such as: electrocardiogram, electrodermal activity, heart rate, skin conductance, and the self-evaluation manikin, among others, have been a part of the analysis objective and systematic of the emotional experience in front of works of art or design; and the semiological analysis presented here for photography, integrates the various results of these experimental studies.

For various reasons, many scholars of semiotics today ignore or overlook new developments in the evolutionary study of human behavior, biology, neuroscience, and perception psychology; as pointed out, for example, by Dr. Wolfgang Tschacher of the University of Switzerland:

Arts experts are commonly skeptical of applying scientific methods to aesthetic experiencing, which remains a field of study predominantly for the humanities. Laboratory research has, however, indicated that artworks may elicit emotional and physiological responses. Yet, this line of aesthetics research has previously suffered from insufficient external validity. (Wolfgang 2016, 1)

In very general terms, semiology studies the way we give meaning to information. There is a long debate about the use of the words Semiology and Semiotics, as well as to what its object of study is. According to its etymology, semiotics carries the suffix "ics" as in mathematics and, according to Gómez de Silva, this means: "from; characteristic of" (Gómez 2013, 358) indicating the condition of "pertaining-to" whatever the noun in the word is. On the other hand, the suffix "logy," as in biology or psychology, corresponds more literally to the thought or study on a subject. In conclusion, we will say that semiology is the science that studies the phenomenon of semiosis (from the Greek semeion=sign and the suffix -sis=action of), that is to say, the process by which a living being that faces a configuration perceived evokes a mental configuration, whereas semiotics is an adjective corresponding to something having a semiosis related quality. In a broad outline, we could say that Semiology is the science that studies semiotics.

Detailing the history of Semiology goes beyond the scope of this document, but I believe it is important to distinguish semiology as an authentic discipline of knowledge, from the non-scientific opinions common today in academia. One of the first record we know of the use of the term semiotics, corresponds to John Locke, who in 1690 published his Essays on Human Understanding, where he is the first to equate semiotics to logic; another of the earliest mentions of semiotics in a disciplinary sense is given in Alexander Baumgarten's Aesthetica Scripsit in 1750; who refers to semiotics as that part of the aesthetics which he treats: "de signis pulcre cogitatorum et dispositorum" (Baumgarten 1750, 4). A concept is developed by him later in his appendix II where he uses both terms—semiology and semiotics—going into detail in the considerations on its object of study. All of this preceded Pierce and Saussure, who were mistakenly considered their founders; none of them coined a term or founded discipline.

Charles Morris published in 1938 "Foundations of the Theory of Signs;" perhaps the first truly semiological work; for it does not inherit the prejudice of confusing the field of study with one of its parts-like Peirce with logic, nor restricting it to a particular sphere-like Saussure with its "within social life" - instead of actual semiology. He assigns to the field its correct and concrete object of study—semiosis, or the phenomenon of meaning — and also contemplates at the same time the whole wide panorama of the semiotic object. 
Men are, among the animals that use signs, the predominant species. Of course, there are other animals that actually respond to certain things as signs of something, but these signs do not reach the complexity and elaboration that we find in speech, writing, art, verification mechanisms, medical diagnosis and signaling instruments own of the humans. (Morris 1994, 23)

Morris is also the first to take seriously the issue of the disciplinarity of semiological study and to consider the need to implement it.

Among the plethora of researchers are linguists, logicians, philosophers, psychologists, biologists, anthropologists, psychopathologists, sociologists and aesthetics specialists. Nevertheless, we do not have a simple but sufficiently global theoretical structure that allows us to incorporate the results obtained from different perspectives and to be able to assemble them into a unified and coherent whole. This study intends to suggest that unifying point of view to which it alluded and to outline the contours of that science of the signs. (Morris 1994, 23-24)

Pierre Guiraud (1971) contributes the notion "semiological functions," base of the development of our approach to the semiological analysis, and Jaime Jiménez Cuanalo, Ph.D., integrates the advances of several sciences to Semiology and postulates several theories. For example, the General Theory of the Meaning, and the Theory of Emotive Reactions (TER), in his work titled "Semiology for Artists and Designers" (2017). Dr. Jiménez refines the concept of semiological functions and formulates the semiologic analysis explained here at. He developed formal—or aesthetic—semiology, based on the advances in the Neurobiologists' view—from authors such as Margaret Livingstone, and psychology of perception-from authors such as R. Arnheim. About the TER, Dr. Jiménez tells us:

outside the modern human being with its arsenal of artificially created concepts and identities, all other living things we know of do not have to interpret the conditions of the world around them as a tool other than their emotional reactions to the different types of configuration. That is to say, all known animals, including our remote or immediate ancestors, have survived because they have an emotional response, an adequate emotion before each common type of configuration. This is called the Theory of Emotive Reactions (TER) that can be stated: Each typical configuration corresponds to a typical emotive response. (Jiménez 2015, 23)

Now then, what is the relationship between TER and hermeneutics in photography? Dr. Jiménez states it as follows:

the meaning of the formal or abstract part of art or design will depend, for the majority of human beings, largely on this system of typical emotive responses to typical configurations. So there will be configurations that will appear appetizing to most humans, or configurations that will appear threatening to most, or depressing; not because of each individual's culture, but simply because of being human. Every effort to express, communicate, or interpret in the context of art and design, has to start from there. In other words, if an artistic or design configuration in photography, painting, or in cinema have formal similarities with the typical configuration of a wildfire, it will be distressing to us, and if it has similarities with the perceptible configuration of the sexual act by the light of the bonfire or of the moon, it will be exciting. Ignoring this foundation of artistic semiotics and design, we may fall into pitiful situations, such as the scene that aimed to be tragic but provoked laughter or attempted to be comical and produced a violent dislike. When this happens, it is not due to the ignorance of the public, because most of them come well equipped with their typical emotional responses, but it is the fault of the artist or designer who has lost the connection with his own intimacy, with his own ancestral emotional responses. (Jiménez 2015, 20)

When we speak of photographic semiotics, we mean the study of the process of meaning between a photographic image and its audience. All photography is mediated by a series of parameters in a composition, thus constituting the formal elements that correspond to the photographer's motive and intention. These codes are the significant agents of photography (framing, point of view, shutter speed, aperture, focal length, color, 
lighting, etc.) that converge in the capture, having light as raw material; the process between the composition of the formal elements fixed by the photographer and their reception by the audience, is what constitutes photographic semiotics.

One of the major problems raised by the semiotic and hermeneutic methods of most authors today, is that their approaches are based mainly on the positions of Barthes, Pierce, or Saussure; and are inadequate to make an objective semiotic analysis on photography since-as we saw earlier-these authors, first, do not clearly define the object of study of semiology or in other cases define it wrong-for example, followers of Roland Barthes make their reflections on the hermeneutics of photography but associated with linguistics and other type of reflections of philosophical and speculative character, making their approaches confusing and impractical.

There are many critics who, fascinated by postmodernists' writing, appeal to the inexistence of limits in the interpretation of photography; museums, exhibitions, galleries, photographic catalogs, etc., are dominated by a critical idealism and moralistic, based on the author's intention, his biography, his cultural context, and the theme of the work, leaving as secondary the analysis of the composition and semiological parameters of photography; a practice that is too widespread today. This document tries to make use of the tools of semiology to uncover the meaning of photographic works.

It must be taken into account that, in order to perform objective hermeneutics on photography, it is important to know, on the one hand, the cultural codification and, on the other, the formal elements and relationships of photographic composition used to produce the image. Umberto Eco clarifies this situation as follows:

Also a visual work of art is a text and as such it has to be examined, and a text is a semantic-pragmatic machine that are perspectives of a semiotics of the visual arts, and demands that it be updated in an interpretative process, and whose rules of generation coincide with their own rules of interpretation, or where the author constructs semiotic artifices by predicting the behaviors of his own addressee, imagining them in advance, inscribing them in the very fibers of his own textual artefact. Therefore, a semiotics of the visual must not be done by analyzing works of painting directly, but starting from the neuropsychological mechanisms of visual perception or-as is being done today-experiments of simulation of the perceptive processes of image recognition by machines intelligent. (Eco 1979, 5)

We start from the hypothesis that, when we experience or observe a photograph of either art or design, two simultaneous readings occur; these are the emotive reading and the conventional reading. Thus, we form a third reading - that is the associative reading — and by these we can decode the total meaning of a photographic work. According to the TER, when we perceive art or design, we are in the same situation as our ancestors faced by the configurations of their environment; except now with artificial configurations added—so we probably will not act upon emotions such as "fight or flight"-but we do present a series of symptoms that express the corresponding emotions, observable in our physiology: such as heart rate, dilation of the pupil, etc. We assign meaning to them along the scale of: good for me, bad for me, or indifferent to my well-being.

\section{The Formal Elements of Photography}

Before proceeding to perform the semiotic analysis—or hermeneutics—in photography, it is important to understand, in general, the way that the photographic language is composed of its formal elements, since they are the ones that make the semiotics of photography possible and therefore make up the meaning of the photographic image. Here is a list of such formal elements. 


\subsection{Frame}

The frame format delimits everything you can express in a photo. All the elements of the photograph are distributed in a frame. Framing is closely related to focal length, and will depend on the angle of vision. The framing of a photograph is the result of the selection of the amount of space included in relation to a "figure," which the photographer decides. The framing in a photograph influences how the viewer perceives the figure; for example, large or small with respect to the background-as in the case of closed or general frames. But it also can make us perceive the subject/object as something solitary, strong, weak, etc. The type of framing is going to generate our perception of a dynamic composition (when it is inclined or vertical), or static (when the shot is made in the line of the horizon), as usually happens with landscape photography.

\subsection{Point of View (or Vantage Point)}

It refers to the position from where the photograph is taken, from where we capture the objective or motif of our image; in a nutshell, it is the angulation in which we take a photo. This can be assessed in respect to the vertical axis or to the horizontal axis - with respect to the object/subject-in which the photograph is made. The point of view correlates the position of the camera, with respect to the subject/object to be photographed. The point of view, in conjunction with the frame, reinforces or magnifies the way we perceive the subject/object: as small, large, isolated, strong, weak. It determines the background of the image, and can even distort subjects/objects, by evoking a sensation of strangeness; for example, in the "nosedive" or "zenith" points of view the subject appears to have a very large head and very small feet—or vice versa, when using the opposite points of view. A ventral point of view, in many cases, gives greater emphasis to the background and causes the image to be viewed from below. We can infer that, as the points of view become extreme, they increase or magnify the effect, since they distort the scene; and because of this, they can create interesting effects, such as the zenith or ventral points of view.

\subsection{The Temporality/Speed}

It related to the shutter speed, is when the photographer makes the decision to freeze movement using high speeds; or makes the flow-of-movement evident, when making shots with low speeds. Taking shots at low speeds results in "sweep," “zooming," "panning," and "mock motion” effects; equally useful—but different-ways to represent that objects in the scene are moving. Semiotically, we can identify that, when we perceive these effects, the emotive connotations of violence, ambiguity, vertigo, continuity, instability, action, dynamism, etc. are reinforced. The effects of the still image will largely depend upon the position of figures within the frame, and their semiotic effects are mostly dependent on the position they hold in reference to the structural skeleton of the frame.

\subsection{Depth of Field}

This is the apparent sharpness range in front of and behind the focus point. Depth of field varies primarily with the lenses' aperture and, in turn, depends on the format and distance of the focus. At the extremes, we have the hyperfocal distance, where everything is in focus, and hypo-focal distance, commonly called low depth of field, that produces an effect called "bokeh.” The semiological aspect of a low depth of field can be related to diffuse, soft, unfocused photographs, and tend to connote a state of dreaminess, memory, hallucination, or some other kind of fiction. In other cases, a lack of sharpness can give the photograph a pictorialist treatment, very 
common among early photographers (as Julia Margaret Cameron, Oscar Gustav Rejlander, Henry Peach Robinson, Gustave Le Gray, etc.). As for high depth of field — where most of the image is sharp, the details or texture are very evident; sometimes the excess of sharpness can have connotations of violence, bluntness, but of course, everything is in relation to the figures and their context or background. The focus control is a technique that allows you to highlight a figure on a background of the image which is very important to mark greater contrast in the image.

\subsection{Focal Length}

It is the distance between the optical center of the lens and the focal point. The focus is the point where the rays of light are concentrated. By varying the focal length, we get a smaller or larger approach; it is what we commonly call "zoom." By modifying the focal length, we change the field of view; so, when we go larger, we seem to get closer, and by reducing it, we appear to move away from the scene. A consequence of this, is to modify the proportion that the objects occupy in the frame. Likewise, it will happen with the background. When we zoom in on an object, the larger portion of the frame it takes the more we are excluding background from the frame. This effect modifies the perspective of objects.

\subsection{Grain or Noise}

We can also call it pixel, noise, or dot. The grain or noise visibility allows us, in many cases, to emphasize the degree of artificial construction of the graphic representation itself. In some cases, it provides the photograph with a pictorial texture connoting dreamlike images, surreal, etc., and in other cases the non-visibility of the grain can perceive an effect of reality. In many cases, journalistic photography is related to photos where the grain or noise is very marked, since the ISO is generally increased.

\subsection{Lighting}

Light is one of the most important compositional elements that can be highlighted in the study of photographic semiotics. There are two types of lighting, natural, and artificial, and these can be soft or hard, thanks to a variety of light modifiers. Rudolf Arnheim considers light as the condition of possibility of the image itself, since it is "a generator of space, and also of time” (Arnheim 1979, 335). Arnheim states that texture is an element at the service of the creation of depth in the image, on which its three-dimensionality depends, in which lighting plays an essential role. We can indicate mainly 3 types of illumination according to the general tone of the image: low key, medium key, and high key. The combination of a tonal key with its light quality, whether it is hard or soft, will also greatly affect the semiotic aspects. If we are faced with a low key lighting combined with hard light, the photo will increase its mystery even to the degree of feeling scary, since their shadows will be denser (little or no tonal information in them). The same happens with the high tone key and hard light, as they are high contrast; clarity, joy, transparency magnifies their stimulus and semiotic response.

\subsection{Structural Skeleton of the Frame}

The analysis of the structural skeleton has its application to the visual arts or works of visual design in general, not only in photography. The importance of this analysis is that it allows us to better understand the visual path in the perceptual field of the image; its rhythm, the distribution of weights, the direction of the subject or objects, which will be very helpful in knowing whether we are facing a dynamic or static photo; 
especially since all photos conform to still-image parameters, only this mechanism allow us to perceive that a subject, for example, is falling or rising in a-photographic — still image. The application of the structural skeleton helps us to visualize if an object is light-ascending, heavy-descending or stable, by determining in which position of the perceptual field it's positioned and if it touches any of the skeletal lines. This will help to know if we are facing a balanced piece or not. Here the vanishing points and perspective are also perceived more magnificently if the point of interest of the image coincides with the center of the photograph. Another parameter that allows us to visualize the skeleton is the identification of the golden ratio, the rule of thirds or dynamic symmetry, fundamental to add interest to the subject/object photographed. We can add that, with the application of the structural skeleton, we can objectively determine if the figures within the image are supposed to move away or approach; its application gives us a key guide to identifying the reason for the clarity or ambiguity in the distribution of the elements in the work and the direction they have. It also helps us to see and identify the planes in an image; in this respect, R. Arnheim points out that: "elements that are grouped by their similarity by a composition tend to be recognized as similar because they are usually on the same plane" (Arnheim 1990).

The use of semiological analysis in the hermeneutics of photography is pertinent because of the materiality of the photographic text. The proposed semiological analysis does not exclude the equal importance of other perspectives for different fields such as sociology or history, but it is important to clarify that the actual meaning of a photograph as art or design is physically coded in the composition, in the relations between its visual elements. This is in contrast to the other hermeneutical perspectives, that focus too much on the biography, the social and cultural context of the artist—like his ideology and religion- to make a semiotic analysis, which can only be an analysis of a historical fact but not of photography itself, generating great confusion when presented as such.

\section{Semiotic Analysis for Photography}

The emotional reading consists on the emotive reactions to a piece, caused by the formal relations between the formal and iconic elements in the composition. In this sense, we can say that the compositional relations in the frame, point of view, shutter speed, aperture, focal length, color and illumination, encode the emotive meaning. We have divided this reading, for its hermeneutic analysis, into two types, the aesthetic function and the phatic function.

- Aesthetic function: the emotive identity of signs: cheerful, hostile, romantic, mysterious, fresh, etc. Or in the figures: heavy, light, dynamic, static, approaching, receding, etc. The aesthetic function has multiple and complex mechanisms and aspects within the formal relations of the composition, such as: tonal-chromatic distribution, geometry, position in space, contrast, background-figure relation, all by the photographic parameters mentioned above, like the framing and point of view.

- Phatic function: mostly primitive communication: "body language,” or formalized or ritualized behaviors, such as religious acts, sporting events, couples during courtship, etc. These are signs—almost symptoms—or applications of signs, for the purpose of generating empathy, sense of belonging or the opposite. The attitude of the characters can reveal irony, sarcasm, be inviting, challenging, rejecting, etc., and promote in the viewer certain types of empathy or antipathy emotions. These attitudes can be studied upon the relationship between the staging and the pose of the subjects in the photograph. The examination of the line of sight of the characters is another aspect that can give us enough clues about the phatic attitudes of the characters. Looking directly 
towards the camera - the viewer — by the subject of the photo, constitutes a direct, challenging interpellation, to the spectator of the image.

Conventional reading refers to what the viewer consciously understands as it is coded in a code the viewer shares with the photographer; is what can be identified and named. It has to do with conventions or social agreements within a culture, and it might be interpreted differently by cultural groups that are not competent in the original code. So that, if you find out which convention is subscribed by the piece, you can know what the author both "says" and "implies." For this, the conventional functions proposed are:

- Denotative function: It consists of "what is meant and said." The receiver of the message must be competent-in and know how to use the code, so that he can understand objectively what the photographer wanted to say and said. Should he be incompetent in using the code, he will probably misinterpret the message, in which case we fall into the connotative function.

- Connotative function: It can be used by the sender of the message when it leaves something implicit or insinuated, but not said or shown; and it is possible for the receiver to decrypt it if he competently applies the code used by the issuer. But there are also rogue connotations that a viewer with a different cultural code or low competency may add by himself but are not part of the objective meaning of the piece.

- Interpretive function: It consists of subsidiary messages that have the sole purpose of helping a receiver to understand the main — different-message. For example, the technical data cards next to works of art in museums, or hand-held programs in the theater.

- Commanding function: It consists of messages that have the direct purpose of causing the receiver to do or stop doing something. We usually identify them as orders, requests, warnings, etc.

- Subjective dimension: It is an aspect of communication where the issuer gives us information about himself in an unintentional way-part of the connotative function; is what he unintentionally tells us about himself, his time, cultural origin, ideology, gender, etc.

\section{Results: Examples of Applied Semiotic Analysis}

We can see in the conventional reading (Figure 1), that what is denoted is two female models, a bottle, some golden bars in the front, a chain in the back and the striped uniforms of the models; which connote that they are in some sort of prison. In the interpretative function, there is no title or additional information, except for the bottle, where we can read a name and percentage of alcohol. That, in addition to its form and typography, conventionally connotes — we associate it — with a bottle of liquor. The image connotes seduction by associating the sexual with the forbidden and dangerous in relation to the models, the staging, and the bottle, where it is suggested or implied that by taking that bottle you will be entering a cell with girls to do "bad things" since the cell symbolizes punishment for some bad deed, and conventionally we know that alcohol acts as a behavior disinhibitor. In the subjective dimension, we can see a tendency of tastes by Caucasian white models, and that probably is or has certain ties with the American culture. 


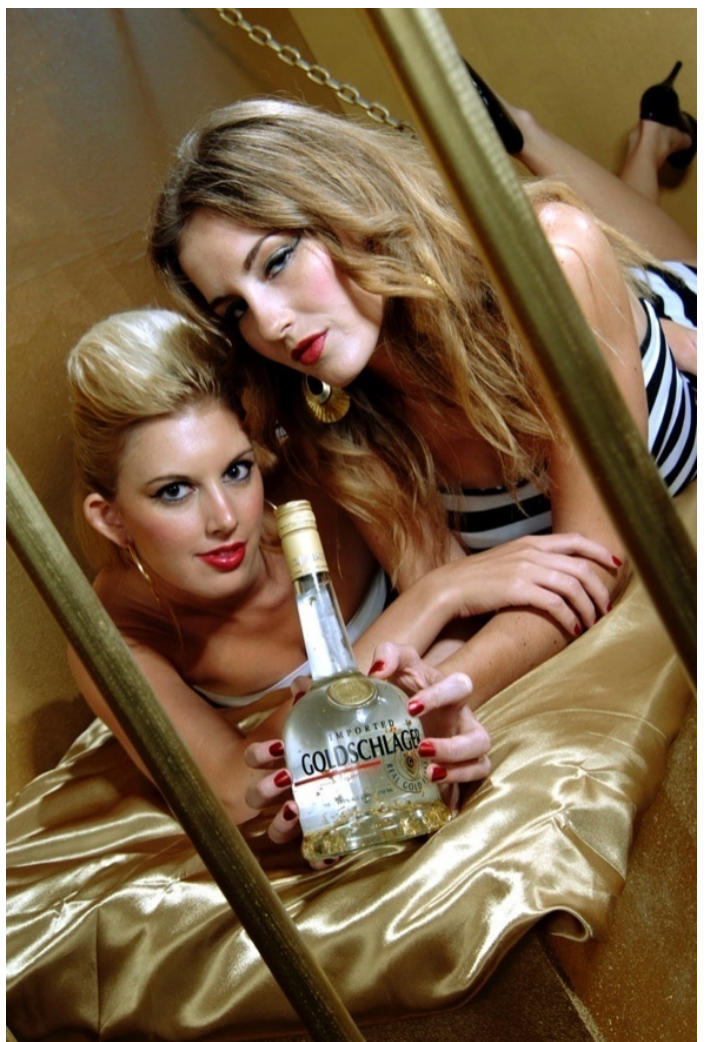

Fig. 1. "Goldschlager” by Salvador Leon. A photo of the "Goldschläger bottle” is a photo that shows two models with a bottle of alcohol in a prison. Taken from: Leon, Salvador (n.d.). Goldschläger bottle [Photograph]. Retrieved from Salvador Leon professional portfolio. Copyright 2008 by Salvador Leon.

In the emotional reading, we can feel a kind of seduction, reinforcing the conventional meaning of the piece: We are called to attention by the golden hue-in nature's semiotics yellow usually entrails a need to pay attention - in the models' hair, the cell, and the bottle of liquor; all this related to the female form of the models, that is biologically encoded the elicit a response. There is a predominating golden color palette accentuated by black, red, white, and blue. We associate combinations of black and red with danger, golden color, on the other hand, it is attractive because of the combination of the organic warm hue with the inorganic shine. It is an attractive composition in the true sense of the word, since the inclination of both heads and part of the models converges in the center, "calling” us inwards towards the composition. The point of view is a nosedive, making the models seem submissive and receptive. In the phatic function, we have the looks of the models—somewhat sexually suggestive-and the position of the feet wearing black heels connoting the idea of game. In the associative reading, we can conclude that we are facing a photograph that means we should get that particular liquor because it is sexy—will associate its owner with inciting women and forbidden games.

Now we have an advertising campaign for a woman's perfume (Figure 2), applying the semiotic analysis in the conventional reading, we have two icons, a model, and a perfume, in the background are all the remaining iconic elements: the chair, the mirror, the crown, and the scepter. In the emotive aspect, we can feel that this image impacts us above all because of the saturated red colors in the objects as well as on the model in her dress - whereas, in nature, saturated usually means chemically active, like nutrient, poisonous or sexually active, and because of the pose of the model—which has a phatic authoritative aspect. The aesthetic semiotic codes emotive identities of authority, force, and imposition. The photographic point of view is of counter nose 
dive, magnifying the coding of authority, because this point of view makes the model seem "larger than life" of "monumental." On the other hand, we face a static composition because the model is in the right hand side of the picture looking left; creating the perception that she is actually posing, we see no indication that she is about to do anything; there is no further narrative. The framing is general-gives us information about the place where the subject is—connoting greatness as it shows her "ruling" over that place. Rudolf Arnheim tells us that the lower right part of the image is the heaviest, and that's where the model sits, placing more emphasis on her and her authority. In the phatic function, the pose is rigid and challenging, evoking the sense of command; in addition, the model is looking directly at the camera, which reinforces her power and authority, as she doesn't shy away from the viewer's gaze. There is a strong tonal contrast between the dress, boots, chair, and the background, which gives the model more sensory impact.

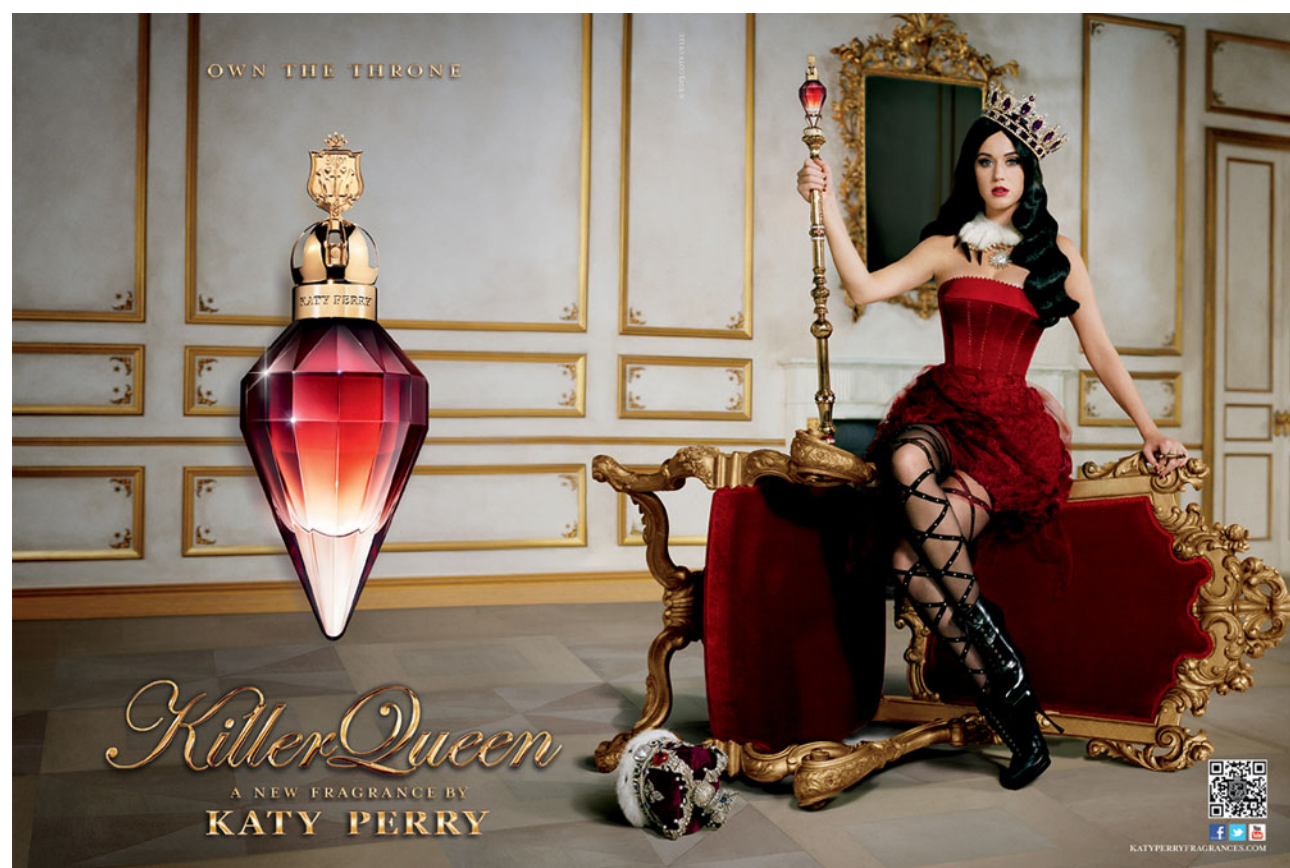

Fig. 2. "Katy Perry Killer Queen Perfume” by Avertex Communications. A photo of the "Katy Perry Killer Queen Perfume.” Killer Queen [Photograph]. Retrieved from Advertex Communications Macys Fall perfume 2013 catalog. Copyright 2013 Macys.

In the conventional reading, we find denotations of a chair, two crowns, a scepter, a mirror, and, judging by the space, a room fit for a queen. In the commanding function, we can read in English a message that says: "own the throne;" which connotes taking charge, suggesting the action of imposing her will. In the interpretative function, we clearly see different messages: "own the throne" and "killer queen, a new fragrance by Katy Perry." This helps us understand the meaning of the image, which refers to the empowerment of aggressive "high-maintenance" women, through the acquisition of the perfume. In the subjective dimension, we can see an idea of the feminine as egotistic empowerment and authority of aggressive women at the expense of man. This is connoted by the fallen crown of a king and the toppled throne where she seats as a conqueror; implying that she defeated the king through the use of the perfume and she is, thus, more powerful. In the associative reading, we can conclude that it means that you should buy Katy Perry's new Killer Queen fragrance, to achieve power and authority over men by using it. 


\section{Conclusions}

As we have seen, thanks to the disciplinary integration of Semiology, it is becoming easier to objectively understand the meaning of the different photographic works that we perceive around us, by applying the exposed semiologic analysis. The importance of knowing and applying the semiologic analysis in the photographic arts and design has its relevance in allowing us to anticipate how our audience will react to the piece that we are going to present and, in this way, to be able to optimize the articulation of the same work, making the necessary adjustments in the composition so that it has the impact we want and can be understood in the way we intended. Be advised that this type of analysis is in its early stages and, therefore, is subject to changes as new data continue to emerge from the various experimental works currently in progress, both by arsology researchers, as well as in the related fields biology, neurobiology, and physiology of vision.

\section{Works Cited}

Arnheim, Rudolf. Arte y percepción visual. Spain: Alianza, 1986.

Baumgarten, Alexander Gottlieb. 1750. Aesthetica Volume 1... Germany: Nabu Press, 2012.

Eco, Umberto. La estructura ausente. Introducción a la semiótica. Spain: Editorial Lumen, 1994.

Gomez, De Silva. Breve diccionario etimológico de la lengua española. México: FCE, 2013.

Guiraud, Pierre. El lenguaje del cuerpo. Mexico: FCE, 2013.

Jiménez Cuanalo, Jaime. Curso de semiología para artistas y diseñadores. Mexico: Zona Límite, 2008.

Morris, Charles. "Foundation Foundations of the Theory of Signs.” International Encyclopaedia of Unified Science. Chicago: University of Chicago Press, 1938.

Kawabata, H. \& Zeki, S. “Neural Correlates of Beauty.” Journal of Neurophysiology 91 (2004): 1699-1705.

Sargent-Pollock, D. N. \& Konecni, V. J. "Evaluative and Skin-Conductance Responses to Renaissance and 20th Century Paintings. Behavior Research Methods \& Instrumentation.” Psychology of Aesthetics, Creativity, and the Arts 9 (1977): 291.

Tschacher, Wolfgang. "Physiological Correlates of Aesthetic Perception of Artworks in a Museum.” Psychology of Aesthetics, Creativity, and the Arts 52 (2011): 2-3. 